\title{
Recommendations for Main Stages Setting-up of Line Distance Relay Protection
}

\author{
Tran Hoang Quang Minh \\ Faculty of Electrical and Electronic Engineering, Ton Duc Thang University, Ho \\ Chi Minh City, Vietnam \\ tranhoangquangminh@tdt.edu.vn
}

\begin{abstract}
In this paper, the technical actions to reduce losses and improve functioning distance relay protection on high-voltage lines is analyzed. Addition to this, criterion technical efficiency of main stages distance relay protection is presented, analyzed and calculated. Based on these analysis and criterion, recommendations for designing and setting main stages distance relay protection are proposed.
\end{abstract}

Keywords: Technical efficiency; technical effect; distance relay protection; electric network; main stage.

\section{Introduction}

The estimation technical efficiency of functioning relay protection (RP) as differences potentially possible effect in the form of an index of faults on protected object and losses (refusals of work, false and excessive actions) are carried to potential effect, is an actual problem for designing and operation RP of an electric equipment and electric networks. Therefore to this question the attention was always paid at the statistical analysis of features of RP work, for example $[1,2,3]$. However there are problems of imposing appearance different components of statistical data. Some data, for example, faults are mass enough, but such events as refusals of operation relay protection at RP, false actions at asynchronous modes, etc. are rather rare. In this connection it is wrongful to use statistical characteristics with different reliability in interesting criteria functional. Therefore there is an actual problem of support statistical adequacy of all components in considered functional. This problem was solved in $[4,5,6,7]$. Solutions of this problem presented in $[8,9,10]$ and in this article this problem is analyzed, used and developed.

\section{Main Part}

On the foundations of the worded definition and the analysis criterion of technical efficiency, and also numerator of this criterion (a difference of potential effect and losses) which is called as technical effect for distance relay protection lines is considered. Losses can be subdivided on three components, which are caused by refusals of equipment, mistake servicing personnel and by functioning relay protection. In the given work last component are considered, which is defined by conditions different topology of a network, modes of sources, switching conditions, types faults, abnormal modes, etc., i.e. different operational conditions. Such choice is made because a number of operational conditions can be changed the operational personnel at use of the same equipment. Hardware refusals depend on manufacturers of element base and devices and mistake servicing personnel depends on the qualification and psychological factors of people. 
In connection with features distance relay protection, consisting in reaction to design parameter resistance from a relay protection installation place on the ends of a line to place fault, which is distributed on the most simple and with final concrete borders uniform PDL on space of each line and other components of the network, is expedient to construct algorithm of technical efficiency with obligatory preservation use of this PDL. This recommendation concerns all steps of distance relay protection, however most simply and unequivocally it is realized at measurements of resistance to places faults on a protected line. At measurement in the fault conditions on previous lines (opposite substations departing from buses) in a direction of distance relay protection action of network elements, it is necessary to consider feeds of place fault from the additional sources connected to opposite substation. And at measurement fault on previous line to the previous components, which are fixed by reserving step distance relay protection, the account of feeds from the sources connected to opposite substations of the previous elements is necessary considered. Under accepted uniform law PDL on all element of the network appears the natural requirement to convert the borders of the stages distance protection lines in coordinate's external (previous and adjacent) elements, that at presence in feeds conditions the border is leaded to different boundary in coordinate these elements. Since in feeds continuously change, borders also change continuously. To account for these changes in the calculation of technical efficiency, need to know the range of these boundaries, especially maximum and minimum values. This can be determined by converting the captured spaces (resistance) of the external elements through the current distribution coefficients between the protected line and the previous lines.

In working or operational modes from which reserving stages should be built up, it is expedient to accept distribution of probabilities resistance normal or close to it since in this case appear met practically in full the main condition normal PDL, notably mass summation commensurable on dispersions of network loadings in the form of full resistance. Thus convention PDL of these resistance for distance protection of each element remains, since electric operating sizes conditions at each element is its. Therefore probabilities of reserve stages false actions are defined as conditional probabilities of acceptance by resistance of gauging of these stages in the range from zero value to borders setting which calculate through setting interesting reserve stage and coefficients current distribution in considered regime-switching conditions of a network (as a rule, maximum and minimum on measured resistance). Similarly conditional probabilities of false actions of all other stages calculate with use normal PDL measured resistance measuring instruments of different stages in working operational modes. As to conditional probabilities of false actions in asynchronous and open-phase modes they are defined also on normal PDL resistance in intervals from zero to borders setting interesting stages, but taking place not in workers, and accordingly in abnormal asynchronous and open-phase modes on a protected line.

Unconditional probabilities of potentially possible effect and losses can be calculated as product of parameters of a stream of interesting events on average duration of action of a corresponding stage of protection, and also as product discussed above conditional probabilities on unconditional probabilities of conditions or network conditions at which they have occurred. These are internal faults on protected object at which there can be operation refusals, faults on external elements concerning protected object when there are excessive actions of considered protection, conditions working operational modes, asynchronous and open-phase modes during which time there are false actions.

Expressions, substantiation, calculation of components of technical effect and technical efficiency are in detail presented in $[8,9,10]$. The subsequent analysis has shown that the technical effect of stages distance protection depends on the parallel and roundabout communications shunting protected, previous, adjacent lines. Depth and switching of these communications leads to change of coefficient current distribution, and the less 
depth (it is more resistance) than the roundabout communication shunting the protected line, the coefficient current distribution for considered distance protection concerning protection of the previous line there is more. At faults on each previous line shunted by roundabout communication, the coefficient current distribution always increases from zero at faults in the beginning of the previous line to some size at faults in the end of this line defined by depth of named roundabout communication. The more the given depth (it is less resistance) than roundabout communication, the more values increases the coefficient current distribution at faults, aspiring to the peripheral ends of the previous lines. Therefore at faults on the previous or adjacent elements depending on a place of damage presence of shunting communications spends to change of borders of grasped areas on these elements not only at the expense of a variation setting, but also and coefficient current distribution. The last complicates definition of probabilities of excessive actions at interaction with protection of the previous (adjacent) elements shunted by roundabout communications.

Also the analysis of a component excessive action in technical efficiency expressions has shown that they take place in case of interaction isochronal stages of protected object and the previous (adjacent) elements, notably at interaction, for example the first stage with the first stages or other high-speed protection of the previous (adjacent) elements, the second stage with the second stages etc.. That is will not be excessive actions at the expense of functioning co-operating stages of protected object occurring at different times and the previous (adjacent) lines, for example, at a variation setting stages of protected object on space of the previous (adjacent) lines in a range from the beginning of these elements in a direction of action co-operating stages to the borders defined settings stages of protection on specified previous (adjacent) lines with smaller time setting in comparison with operating times co-operating protection on protected object. The last allows asserting that at the expense of interaction of not high-speed stages of lines protection with differential protection of the previous elements then functional excessive actions of the specified steps essentially cannot be at faults on all space of the previous elements. Therefore in the further analysis it is possible to exclude transformer elements which are always equipped by differential protection from consideration.

The given analysis shows that at a variation setting stages distance protection technical efficiency at the basic stages has a maximum on the distant (opposite) end of a protected line or the beginning of the previous (adjacent) lines since at faults in this point is not present either operation refusals, or excessive actions. False actions which define decrease in technical efficiency concerning unit, its maximum positive value take place only. And this decrease more than measuring instruments are more sensitive, i.e. than it is more resistance setting. Similarly at reserve stages natural maximum positive value of technical efficiency takes place on border of reserved lines and departing from them previous to previous (adjacent) lines.

However and accordingly optimum settings stages it is possible to take advantage of the given maxima of technical efficiency only in case of the first stage. Other basic stages with great time settings formally have the same optimum value setting, but for them such setting is senseless, since they should provide reliable protection of the end of a line, i.e. with the guaranteed factor of sensitivity so their settings spaces of the previous lines should be carried deep into. Even more useless though also explainable will be settings the given stages if them to move on space of a protected line at which there will be refusals. It is similarly possible to tell and about reserving stages at private basic analysis stages of their efficiency by consideration of work of a protected line through a concrete previous (adjacent) line on the previous elements to the previous (adjacent) line, i.e. elements of the second periphery. In other words, the maximum of technical efficiency in this case takes place on border of the opposite end of the previous line and the beginning of lines of the second periphery. However setting of reserving stage should guarantee reliable protection at faults on the remote end of the previous (adjacent) line, i.e. to satisfy 
demanded minimum sensitivity. Therefore setting should be displaced on space of elements of the second periphery for achievement of the minimum sensitivity. Moving setting of reserving stage on the previous line as is senseless as well as setting the basic stages with endurance of time for a protected line.

Thus, though functional refusals of operation can be at all stages, the logic of adjustment of all steps doesn't suppose use of such adjustment as actually senseless. Decrease in technical efficiency at the expense of false actions always takes place and increases in process of sensitivity increase (resistance setting increase) stages on all range of response parameter. Thus functional refusals at these sages are absent, and excessive actions or also are absent on sites of response parameter resistance occurring at different times, or take place on isochronal sites of interaction of stages of protected and previous (adjacent) lines at faults on the last. Excessive actions at faults on specified isochronal sites in addition reduce technical efficiency. However it takes place for high-speed (without time setting) the first stages, setting which on resistance appear on space of the previous lines. Functional excessive actions of stages with time setting for commensurable on length protected and the previous lines appear excluded thanks to the joint coordination on response parameter and time of stages of a protected line with more high-speed stages of the previous lines. In case of short previous lines it is not possible to carry out the given joint coordination owing to necessity to provide sensitivity of sages with time setting of a protected line. Therefore arise isochronal sites of resistance on the previous short lines, localized from borders of more high-speed stages of the previous lines to the opposite ends of the last or the ends of steps of a protected line. At faults on the specified sites there are in common actions isochronal stages of a protected and previous short line or refusal of a step of a short line which are qualified as excessive actions of steps of a protected line: In the first case in half of cases, and in the second - all refusals of actions of a stage of the previous short line. Highlights of the analysis are displayed in Figure 1.
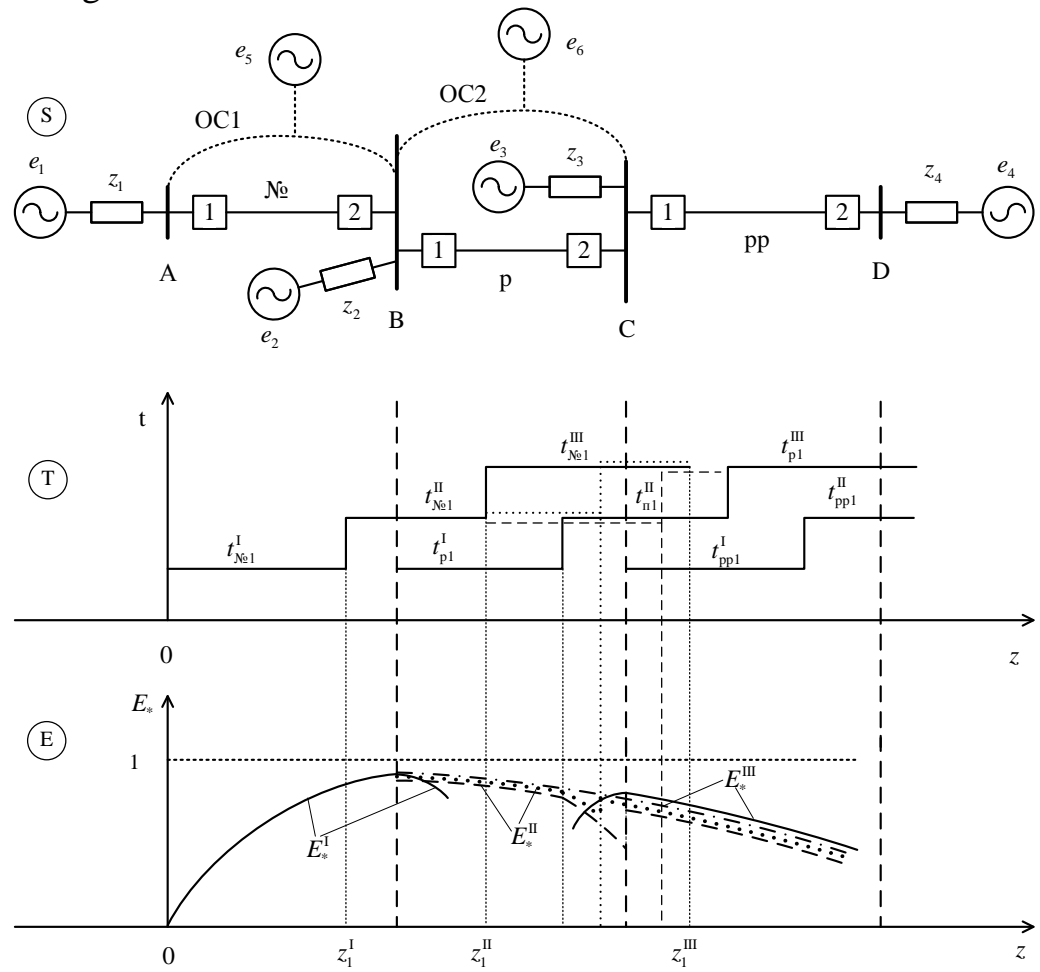

Figure 1. Network Structure (S), Action Times (T), Technical Efficiency (E) of Distance Protection № 1 on line №. 
Network structure (S), action times (T), technical efficiency (E) of distance protection taking into account noted features it is illustrated on figure 1 , where indicated:

A, B, C, D - substations of a network,

№, $\mathrm{p}, \mathrm{pp}$ - protected (№), previous (p), previous to previous ( $\mathrm{pp}$ ) lines of a network,

$\mathrm{OC} 1, \mathrm{OC} 2$ - the roundabout communications shunting accordingly protected (№) and previous (p) lines,

$e_{1} \ldots e_{6}-$ sources,

$z_{1} \ldots z_{4}-$ resistance of sources,

Numbers 1, 2 in rectangles - a designation of switches or complete protection on the ends of lines,

№ $1, \mathrm{p} 1$, pp 1 - line protection numbers of at acting directions of considered protection № 1 ,

$z_{\text {No1 } 1}^{\mathrm{I}}, z_{\mathrm{N} 01}^{\mathrm{II}}, z_{\mathrm{N} 01}^{\mathrm{III}}$ - resistance setting of the I (first), II (second), III (third) stages of distance protection № 1 ,

$t_{\text {№1 } 1}^{\mathrm{I}}, t_{\mathrm{N} 101}^{\mathrm{II}}, t_{\mathrm{N} \mathrm{N} 1}^{\mathrm{III}}-$ time setting of the I (first), II (second), III (third) stages of distance protection № 1, are similarly designated time setting of other stages and protections, for example $t_{\mathrm{p} 1}^{\mathrm{II}}$,

$E_{*}^{\mathrm{I}}, E_{*}^{\mathrm{II}}, E_{*}^{\mathrm{III}}-$ curves of technical efficiency of the I (first), II (second), III (third) stages of protection № 1 .

It is visible that the curve of efficiency of the first stage $E_{*}^{\mathrm{I}}$ distance protection № 1 at change setting around the opposite end line has a maximum. The analysis shows that this maximum in the conditions of the account only functional losses takes place on border of protected and previous lines since thus is not present either operation refusals, or excessive actions. And the size of technical efficiency in it to a point is lowered concerning unit on the relation of probability of false actions to potentially possible effect $\mathrm{p}$ (A). Technical efficiency of the second stage $E_{*}^{\mathrm{II}}$ of considered protection within a range of interaction occurring at different times with the first stage previous (p) lines at faults on it can decrease only since operation refusals in these conditions essentially are not present, and excessive actions also is absent owing to time blocking of action of a considered second stage at faults on the previous line [3]. As to false actions they in process of increase in sensitivity of the second stage (increase in resistance of operation) increase. The last leads to decrease technical efficiency $E_{*}^{\mathrm{II}}$ (solid line and the curve in Figure 1).

At the further increase in sensitivity (the resistance setting increase) setting of second stage can get to a range isochronism actions with the second stage of the previous line protection. It will cause occurrence of excessive actions of considered protection № 1 that leads to more intensive decrease in efficiency of its second stage $E_{*}^{\mathrm{II}}$ (a line from points and a dot-dash line). And it will take place, while setting value will not get on a line of following more distant periphery. Then interaction of the second stage of considered protection will be carried out with the first stages of the second periphery occurring at different times that will not give the contribution to excessive actions of the considered protection second stage. Hence, technical efficiency $E_{*}^{\mathrm{II}}$ will monotonously decrease at faults on all space of the previous lines with intensity increase on isochronal sites with a hyper sensibility while value of the second stage of considered protection will not get on sites of the second periphery. In other words, technical efficiency of the second stage monotonously decreases at the expense of false actions on space of the previous lines, and at occurrence isochronal sites for the last decreases more strongly up to the end of the previous lines. 
The time setting characteristic of the third stage of a protected line as the basic, i.e. adjusted by the coordination with the second stages of the previous elements (continuous lines on fig. 1) is that isochronal sites on space of the previous line are not present. Therefore the curve of technical efficiency $E_{*}^{\mathrm{III}}$ of the third stage as the basic does not contain the jumps caused by excessive actions, brought faults on isochronal sites, and continuously decreases because of losses only false actions (a dot-dash line with two intermediate points). The maximum value curve both the first, and the second stages settles down in the end of a protected line.

The time setting characteristic of the third stage of a protected line as reserve, i.e. adjusted by an independent choice setting on response parameter (resistance), the obligatory coordination on time with reserve stages of the previous lines and the possible coordination on response parameter with reserve stages of the last does not contain in an opportunity of radial structure of a network isochronal sites on lines of the second periphery and, hence, does not cause the strengthened decrease in curve technical efficiency at the expense of excessive actions at faults on spaces of these lines (a continuous one-topmost curve fig. 1 around the remote end of the previous line). However at faults on the previous line owing to resistance reduction setting or higher last the curve of technical efficiency cannot increase according to disappearance excessive and decrease in probabilities of false actions, since quantities of refusals of operation arise and increase. The curve of technical efficiency can increase, but to a lesser degree because of operation refusals, and can and decrease in connection with essential increase in quantity of refusals of operation.

The analysis shows that in the majority of practical cases protected and external lines in a acting direction of protection have commensurable lengths that allows setting to the second stage to co-ordinate reliably with the first stage of all previous lines and to provide thus sufficient sensitivity. At the given set-up losses of functioning excessive actions is not present. But in the presence of short previous lines such set-up leads deepening to the second stage, up to loss of demanded minimum sensitivity. The usual decision in the presence of short lines consists in elimination of the coordination with the first stage of short lines. It is reached by application for short lines differential protection that provides independence of functioning protection of short lines with stages protection of a network. However differential protection of lines is rather expensive because of the demanded interaction channel on the ends of short lines. At the same time not the account of short lines at the coordination of stages protection leads to insignificant decrease in technical efficiency because of excessive actions at faults on space of these lines, between setting their first stages and the end of these lines. In this connection it is possible to recommend set-up of the second stage of protected lines at presence on short previous lines of usual stages relay protection. Thus definition of technical efficiency criterion of a considered protection second stage as quantitative guarantor of quality of applied protection is obligatory. In the given criterion along with the general loss of false actions in operational workers, abnormal asynchronous and open-phase modes, excessive actions should be considered at faults on all short previous lines.

Designing procedure in this connection can be simplified and reduced. The first stage setting can it is accepted a protected line equal to resistance and at this setting to define size of technical efficiency in the conditions of the account only losses of false actions. It is possible to define also size of technical efficiency in the conditions of a positive error of response parameter, causing excessive actions, also in the conditions of a negative error of response parameter, causing operation refusals. Setting on resistance it is expedient to second stage to define, proceeding from sensitivity at faults in the end of a protected line, extensive and routine coordination with the first stages of the previous lines proportional to the protected line, completely to exclude, count up losses of excessive actions for the account isochronism the second stages on short previous and protected lines which sum up with losses of false actions, to find in whole technical efficiency of the second step of a 
protected line as in the conditions of absence of an error of response parameter, and presence of these errors. Setting on resistance at each private basic analysis stage of their efficiency it is expedient to third reserving stage to define, proceeding from sensitivity at faults in the end of the previous line, extensive and routine coordination with any stages of lines of the second periphery proportional to protected and previous lines, completely to exclude, calculate losses of excessive actions for the account isochronism the second stages on short and protected lines which sum up with losses of false actions, to find in whole technical efficiency of the second stage of a protected line as in the conditions of absence of an error of parameter, and presence of these errors.

Expressions of criteria technical effect and its components are given below:

1. For first zone (stage) of distance relay protection:

$$
E_{\text {№1 }}^{\mathrm{I}}=\mathrm{p}\left(\mathrm{A}_{N_{\mathrm{No}}}^{\mathrm{I}}\right)-\mathrm{p}\left(\mathrm{O}_{\text {№1 }}^{\mathrm{I}}\right)-\sum_{1 \mathrm{pi}=1}^{n_{\mathrm{p}}} \mathrm{p}\left(\mathrm{U}_{\text {№1-1 pi }}^{\mathrm{I}}\right)
$$

2. For second zone (stage) of distance relay protection:

$$
E_{\text {№1 }}^{\mathrm{II}}=\mathrm{p}\left(\mathrm{A}_{\text {№ }}^{\mathrm{II}}\right)-\mathrm{p}\left(\mathrm{O}_{\text {№1 }}^{\mathrm{II}}\right)-\sum_{1 \mathrm{pi}=1}^{n_{\mathrm{p}}} \mathrm{p}\left(И_{\text {№1-1 pi }}^{\mathrm{II}}\right)
$$

Where the lower indexes: № - protected line, $\mathrm{p}$ - previous lines (elements), pp previous (elements) of previous lines (elements) (Fig. 1). The letter $\mathrm{p}$ designates probabilities of events: A - faults on a protected line, $\mathrm{O}$ - operation refusals, И excessive actions; I - first stage, II - second stage, III - back-up stages of line distance relay protection.

3. Probabilities faults $\mathrm{p}(\mathrm{A})$ :

3.1 For the first stage

$$
\mathrm{p}\left(\mathrm{A}_{\text {№ }}^{\mathrm{I}}\right)=\omega_{\text {№ }} \mathrm{m}\left(T_{\text {№ }}^{\mathrm{I}}\right)
$$

3.2 For the second stage

$$
\mathrm{p}\left(\mathrm{A}_{\text {№ }}^{\mathrm{II}}\right)=\omega_{\text {№ }} \mathrm{m}\left(T_{\text {№ }}^{\mathrm{II}}\right)
$$

(6)

Where $\omega_{\text {ํo }}-$ the flow parameter of interest fault types on the protected lines, $\mathrm{m}\left(T_{\text {№ }}^{\mathrm{I}}\right)$ и $\mathrm{m}\left(T_{\text {№ }}^{\mathrm{II}}\right)$ - average duration of detection (lock) fault channels of the first and second stages (substantially the setting time of the first and second stages).

\section{Excessive actions}

Excessive actions of the first stage take place in the external fault:

1) As a joint action with isochronous speed protections (the first stage, the protection on the differential principle) for faults on previous lines,

2) As refusals of the first stages on the previous lines.

Proceeding from the above, to the probability of excessive action of the first stage of the protected line should be show below:

$$
\sum_{1 \mathrm{pi}=1}^{n_{\mathrm{p}}} \mathrm{p}\left(\mathrm{U}_{\text {№l-1pi }}^{\mathrm{I}}\right)=\sum_{1 \mathrm{pi}=1}^{n_{\mathrm{p}}}\left[\frac{1}{2} \mathrm{p}\left(\text { №lpi }_{\text {№ }} / \mathrm{BK}_{1 \mathrm{pi}}\right) \mathrm{p}\left(\mathrm{BK}_{1 \mathrm{pi}}\right)+\mathrm{p}\left(\mathrm{O}_{\text {№l } \mathrm{pi}} / \mathrm{BK}_{1 p i}\right) \mathrm{p}\left(\mathrm{BK}_{1 p i}\right)\right]
$$


(8)

Where Д, O - joint action, refusals of protection for the 1pi-th elements, BК - faults on the 1pi-th elements.

A numerical results with using the developed algorithms are shown below on the example of the calculation and analysis of distance relay protection line $220 \mathrm{kV}$ Substation Surgust - Substation Contur (Distance relay protection on side of the substation Surgust) on one of the Russian power system. The topology of the analyzed area is shown in Fig. 4. Line p1, p2 and p3 are previous lines (the first periphery); pp1 and pp2 lines are lines of second peripheral.

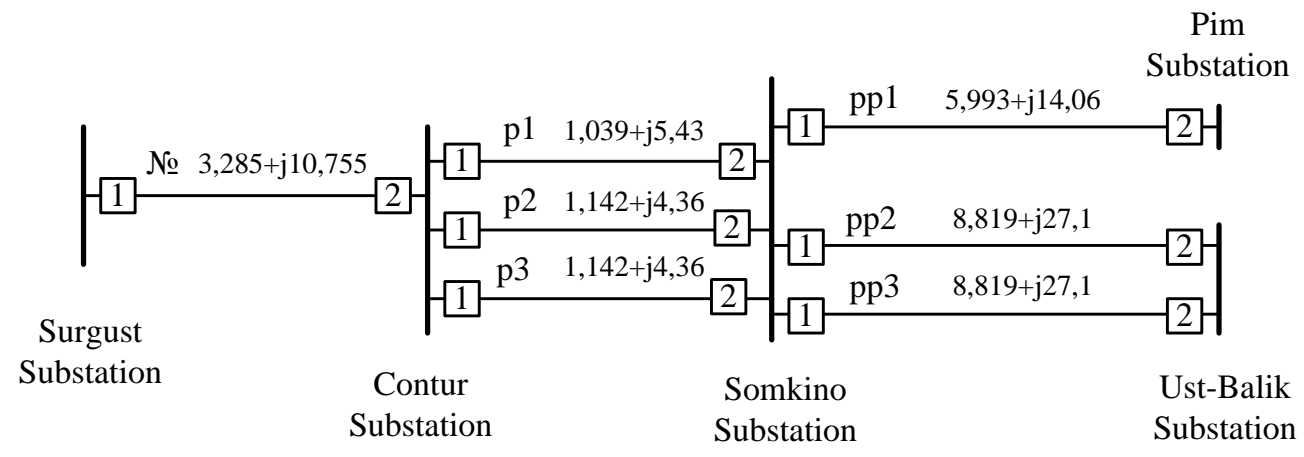

Figure 2. The Topology of the Analyzed Area

The settings of second stages of the distance relay protection on the lines p1, p2, p3 are chosen by the guidelines [1-3]. For the considered № 1 distance relay protection №,1, setting the second stage are based on the high technical efficiency at the opposite end of the line №. The results of calculation technical efficiency by varying the settings are presented in the tables 1 .

Table 1. The Numerical Results of Technical Efficiency by Varying the Setting of the Second Stage Distance Relay Protection №,1

\begin{tabular}{|c|c|c|c|}
\hline $\begin{array}{c}\text { Setting of the } \\
\text { second stage } \\
\text { (Ohm) }\end{array}$ & $\begin{array}{c}\text { Probability of } \\
\text { refusals }\end{array}$ & $\begin{array}{c}\text { Probability } \\
\text { of excessive } \\
\text { actions }\end{array}$ & $\begin{array}{c}\text { Technical } \\
\text { efficiency (\%) }\end{array}$ \\
\hline 14,048 & 0 & 0 & 99,6 \\
\hline 14,5 & 0 & 0 & 99,44 \\
\hline 15 & 0 & 0 & 99,16 \\
\hline 15,5 & 0 & $0,25.10^{-10}$ & 96,22 \\
\hline 16 & 0 & $0,559.10^{-10}$ & 91,55 \\
\hline 16,5 & 0 & $1.10^{-10}$ & 84,9 \\
\hline 17 & 0 & $1,44.10^{-10}$ & 78,26 \\
\hline
\end{tabular}




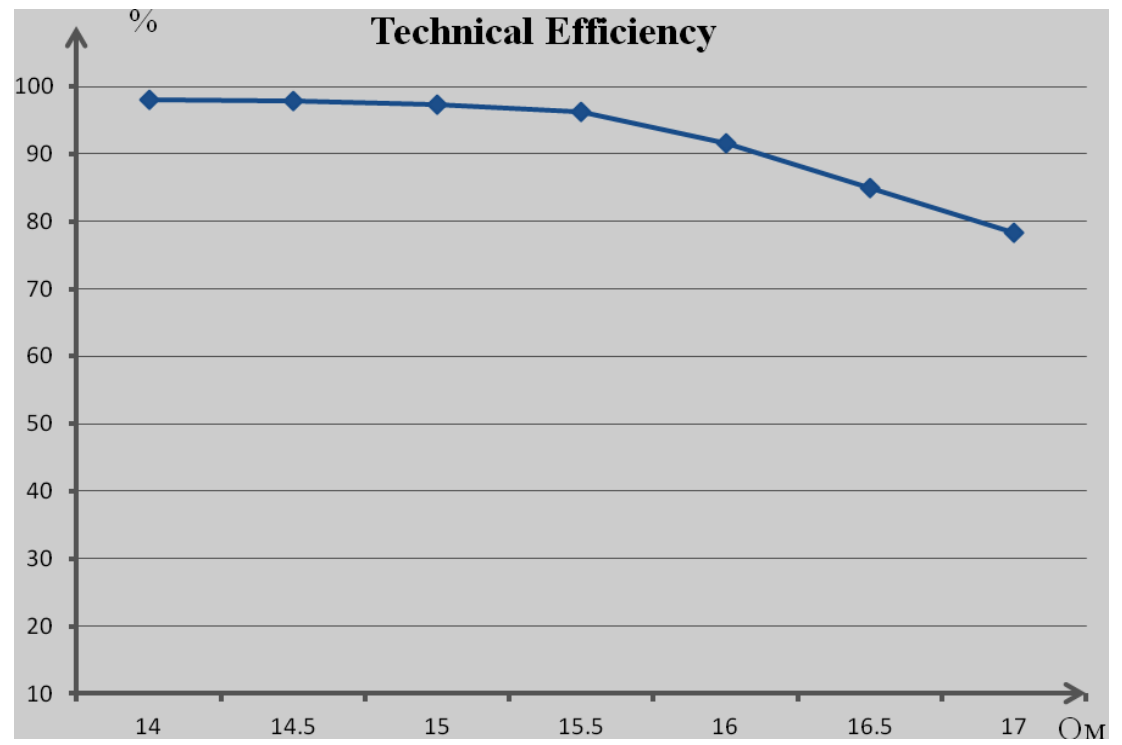

\section{Figure 3. Technical Efficiency of Second Stage (Distance Relay Protection of High-voltage Line)}

The numerical results under specific restrictions confirm derived from the phenomenological analysis of the findings of the maximum technical efficiency and its changes. It is evident that excessive actions in the second stage there are no set-point until it reaches the end of the first stages of previous lines and will not begin the second stage of action isochronous protect the protected line № 1 with the second stage of the protection $\mathrm{p} 11, \mathrm{p} 21, \mathrm{p} 31$ previous lines $\mathrm{p} 1, \mathrm{p} 2$ and $\mathrm{p} 3$. With these results, may be recommending:1) Increase setting of the first stage to the resistance equal to resistance of all line or to near this value;2) Setting of the second and third stages with the minimum of excessive actions, proceeding from their sensitivity. Positive values of technical efficiency, which are near to the one unit, are the highest technical quality of distance relay protection.

\section{Conclusion}

The presented technical efficiency analysis of line distance protection stages allows:

- To recommend increase setting of the first stage to the resistance equal to resistance of all line,

- To prove absence of functional excessive actions of the basic stages with time setting, also reserving stages at faults on external elements concerning their protected zones within borders of protection stages on external elements with smaller times of action,

- To recommend setting of the basic stages with time setting, also reserving stages, proceeding from their sensitivity.

\section{References}

[1] A. M. Fedoseev, "Relay protections power system", M.:Energoatomizdat, (1984), p.520.

[2] E. M. Schneerson, Digital relay protection, Energoatomizdat, (2007), p. 549.

[3] G. S. Nudelman and A. I. Shalin, "Microprocessor-based relay protection", News of electrical engineering. (2008), pp. 74-79.

[4] A. V. Shmoilov, "Probability technologies in electric power industry", Proc. 6-th Russian-Korean Intern. Symp. on Science and Technology KORUS, Novosibirsk, vol. 2, (2002), pp. 421-424. 
[5] A. V. Shmoilov, L. V. Krivova, E. I. Stoyanov and K. V. Ignatiev, "Probabilistic method select of the borders interval data for electroenergetic problems", Proc. The High School, Problems of energy, no 7-8, (2008), pp. 146-157.

[6] A. V. Shmoilov, "Probability technologies in electric power industry", Proc. 6-th Russian-Korean Intern. Symp. on Science and Technology KORUS, vol. 2, (2002), pp. 421-424.

[7] A. F. Prutik and A. V. Shmoilov, "Setting-up algorithms of relay protection", The Forth International Forum on Strategic Technology (IFOST), (2009), pp. 45-50.

[8] A. F. Prutik, T. Minh and A. V. Shmoilov, "The selectivity and the technical efficiency of relay protection and automatics”, Journal Energy problems, (2010), pp. 154-163.

[9] T. Hoang, Q. Minh and A. V. Shmoilov, "Technical efficiency of line distance relay protection", The Fifth International Forum on Strategic Technology (IFOST), (2010), pp. 335-340.

[10] T. Hoang, Q. Minh and N. T. Thang, "Criterion Technical Efficiency of Line Distance Relay Protection", International Journal of Advanced Science and Technology, vol. 53, (2013), pp. 123-129.

\section{Author}

Tran Hoang Quang Minh defended his PhD thesis at Tomsk Polytechnic University, Tomsk City, Russian Federation. The author's major fields of study are High-voltage Power System and Relay Protections. He is working as Lecturer in Faculty of Electrical and Electronic Engineering, Ton Duc Thang University, Ho Chi Minh City, Vietnam. 